\title{
Fast single-photon avalanche diode arrays for laser Raman spectroscopy
}

\author{
Jordana Blacksberg, ${ }^{1, *}$ Yuki Maruyama, ${ }^{2}$ Edoardo Charbon, ${ }^{2}$ and George R. Rossman ${ }^{3}$ \\ ${ }^{1}$ Jet Propulsion Laboratory, California Institute of Technology, Pasadena, California 91109, USA \\ ${ }^{2}$ Circuits and Systems, Delft University of Technology, Delft 2628CD, The Netherlands \\ ${ }^{3}$ Division of Geological and Planetary Sciences, California Institute of Technology, Pasadena, California 91125, USA \\ *Corresponding author: jordana.blacksberg@jpl.nasa.gov
}

Received June 30, 2011; revised July 26, 2011; accepted August 21, 2011;

posted August 22, 2011 (Doc. ID 150202); published September 14, 2011

\begin{abstract}
We incorporate newly developed solid-state detector technology into time-resolved laser Raman spectroscopy, demonstrating the ability to distinguish spectra from Raman and fluorescence processes. As a proof of concept, we show fluorescence rejection on highly fluorescent mineral samples willemite and spodumene using a $128 \times 128$ single-photon avalanche diode (SPAD) array with a measured photon detection efficiency of $5 \%$. The sensitivity achieved in this new instrument architecture is comparable to the sensitivity of a technically more complicated system using a traditional photocathode-based imager. By increasing the SPAD active area and improving coupling efficiency, we expect further improvements in sensitivity by over an order of magnitude. We discuss the relevance of these results to in situ planetary instruments, where size, weight, power, and radiation hardness are of prime concern. The potential large-scale manufacturability of silicon SPAD arrays makes them prime candidates for future portable and in situ Raman instruments spanning numerous applications where fluorescence interference is problematic. (c) 2011 Optical Society of America

OCIS codes: $\quad 300.6450,300.6500,040.1345,040.6070$.
\end{abstract}

Raman spectroscopy is a nondestructive technique with the unique ability to provide structural and compositional information for nearly all forms of matter. As the technique has become ever more sensitive, previously inaccessible applications have emerged, for example, in nanomaterials, art and archaeology, biological and medical sciences, and planetary science [1-7]. One issue that all of these fields have in common is interference from unwanted background fluorescence, which is often much larger than the Raman signatures of interest. An effective solution is time-resolved Raman spectroscopy, which can detect Raman signatures under all fluorescence conditions. Since Raman emission is virtually instantaneous, while fluorescence occurs over longer time scales, a fast time-resolved detector can be used to distinguish spectra from both processes.

Using highly fluorescent Mars analog minerals, we have previously reported on the potential value of time-resolved Raman spectroscopy applied to in situ planetary mineralogy [8]. Using a pulsed laser and a streak camera, we obtained spectra where the traditional $\mathrm{CW}$ green laser approach fails due to overwhelming fluorescence. These results are particularly significant for altered minerals, which can have very large fluorescence and are often of the greatest interest.

In order to minimize fluorescence interference, the majority of commercial and handheld portable Raman spectrometers presently on the market use near-IR laser excitation and sometimes dual wavelengths (532 and $785 \mathrm{~nm}$ ). Though the use of a longer-wavelength source is effective in many cases, it suffers from a lowerintensity Raman return compared to excitation at $532 \mathrm{~nm}$. Time-resolved Raman is an alternative approach that has not been used commercially in large part due to the cost, size, and complexity of detectors with adequate time response, namely, traditional photocathodebased imagers such as intensified CCDs and streak cameras. For specialized applications, photocathode-based systems have been shown to very effectively minimize fluorescence interference, for example in remote Raman instruments under development for planetary mineralogy [9].

Until recently, a solid-state alternative was deemed out of reach due to limitations in combined detector format, bandwidth, and sensitivity. But the recent advent of compact and low-power silicon single-photon-avalanchediode-(SPAD)-based sensors with the potential for largescale manufacturability can enable a new generation of high temporal resolution imaging Raman spectrometers. This development is particularly relevant to in situ planetary spectroscopy since it offers a significant reduction in size, weight, power, and overall complexity, putting it on par with instruments that do not have time resolution, but providing enhanced science return. Additionally, SPADs have been shown to be particularly radiation hard [10], a necessity for space applications.

Here we demonstrate that time-resolved Raman is now achievable using an all-solid-state detector array-a $128 \times 128 \mathrm{SPAD}$ - as an alternative to the streak camera (Fig. 1). We also propose that, with straightforward modifications to the present SPAD design, we can expect to achieve improved sensitivity over the streak camera.

The $128 \times 128 \mathrm{SPAD}$ array used in this Letter (designed at Delft University of Technology and detailed elsewhere [11]) was originally designed for on-chip fluorescence detection and fluorescence lifetime imaging microscopy (FLIM). The effective fill factor is $4.5 \%$, and a microlens array is used with a concentration factor of 1.59. Though not ideally designed for Raman spectroscopy, this chip was sufficient for demonstrating the feasibility of the approach. Future work will focus on tailoring the chip to our application for improved performance.

The laser Raman setup is the same as reported in [] with the following exceptions. For these experiments, we use a Teem Photonics pulsed microchip laser with $1 \mu \mathrm{J} /$ pulse running at $40 \mathrm{KHz}$. The SPAD is synchronized 

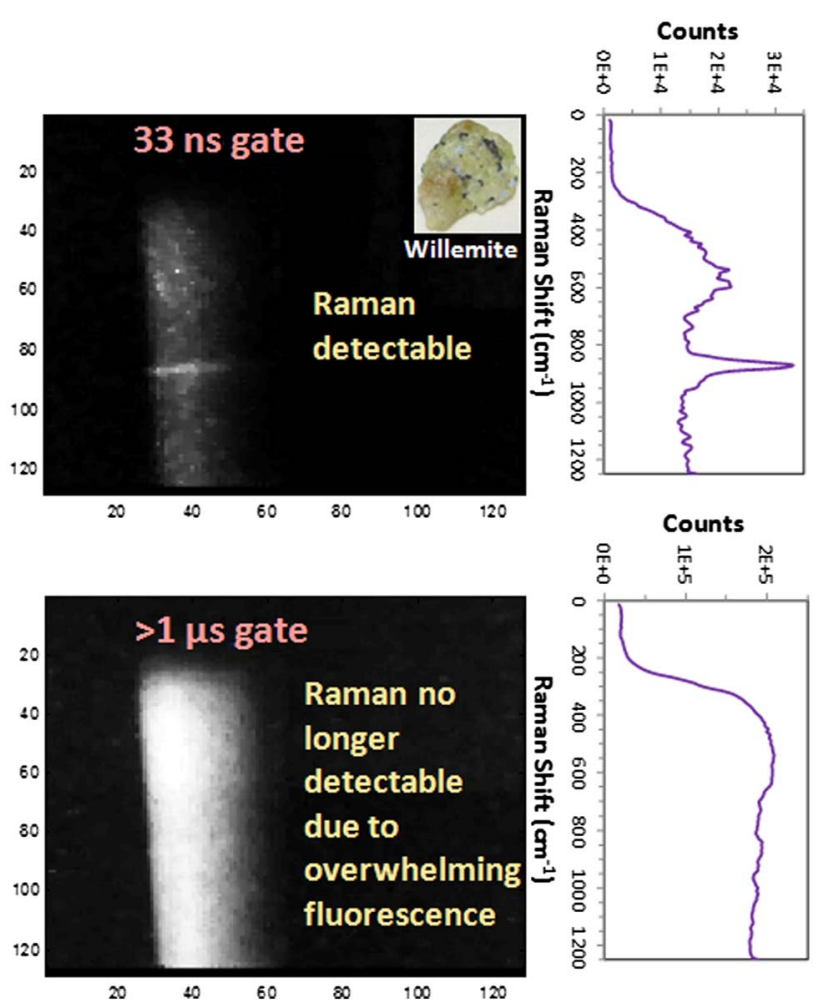

Fig. 1. (Color online) Time-resolved spectra of the highly fluorescent mineral willemite using the $128 \times 128 \mathrm{SPAD}$ array. The top image uses a shorter gate time, eliminating the large fluorescence background. The spectra on the right are generated by summing 30 columns in the image.

with the laser using a single-pixel SPAD trigger chip. For demonstration purposes a broadband grating (spectrometer resolution $\sim 10 \mathrm{~cm}^{-1}$ ) was used instead of a highresolution Raman grating (spectrometer resolution $\sim 1 \mathrm{~cm}^{-1}$ ) in order to image a sizable portion of the Raman spectrum on the SPAD array, which is only $3.2 \mathrm{~mm}$ wide with $25 \mu \mathrm{m} \times 25 \mu \mathrm{m}$ pixels. In the future the array can be made larger and the spectral resolution and sensitivity improved. Experimental parameters are listed in Table 1.

We determined the sensitivity of the SPAD array in our setup by measuring the laser power focused on the SPAD with the notch filter removed. Laser power was measured with a National Institute of Standards and Technology calibrated photodiode, and neutral density filters were used in the beam path to vary the incident power. The mean number of counts in a frame, as a function of the number of incident photons in a frame, was then determined (Fig. 2). The slope of this plot yields a photon

Table 1. Experimental Parameters
Laser wavelength
Laser pulse energy
Laser pulse width
Laser repetition rate
Chip speed
Time per frame
Frames per measurement
SPAD on time
SPAD breakdown voltage
SPAD excess bias
SPAD DCR
$532 \mathrm{~nm}$
$1 \mu \mathrm{J} /$ pulse
500 ps
$40 \mathrm{KHz}$
$40 \mathrm{MHz}$
$409 \mu \mathrm{s}$
10,000 (typical)
$7.03 \mathrm{~ns}$
$19.1 \mathrm{~V}$
$5 \mathrm{~V}$
$1830 \mathrm{~Hz}$ (at $5 \mathrm{~V}$ )

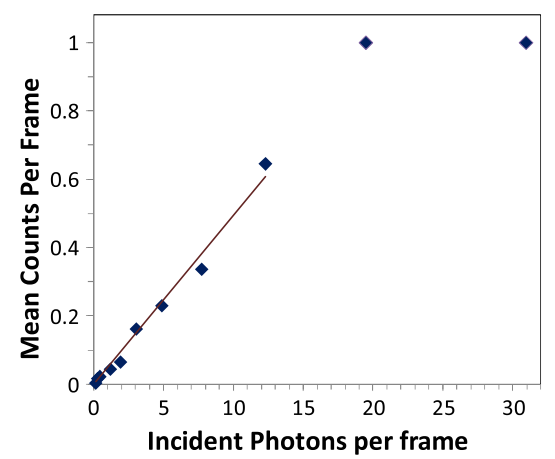

Fig. 2. (Color online) Counts per frame as a function of the number of photons incident on the SPAD. The PDE obtained from the curve fit is $5 \%$.

detection efficiency (PDE) of 5\%. Given the fill factor and microlens concentration factor, this translates into a photon detection probability of $70 \%$. We expect that the PDE can be improved by over an order of magnitude by increasing the fill factor and correcting an error in the fabrication of the microlens array, which caused almost a factor of 10 reduction in the concentration factor. We have future plans to pursue these improvements. It should also be noted that the dark count rate (DCR) of the SPAD is extremely low $(1830 \mathrm{~Hz}$ at $5 \mathrm{~V}$ excess bias). When considering that the SPAD is only active for $7 \mathrm{~ns}$ at a time, if the SPAD is synchronized with the $40 \mathrm{KHz}$ laser (and not free running at $40 \mathrm{MHz}$ as it is in the current chip), each frame would contain $\sim 10^{-4}$ dark counts. At this low level the DCR has almost no impact on sensitivity, even if the active pixel area were increased.

In comparing the results to those obtained with our streak camera system (which uses a PHOTONIS P925 streak tube), the PDE can be compared to the quantum efficiency (QE) of the photocathode, which increases from $5 \%$ to $10 \%$ from 620 to $532 \mathrm{~nm}$. At longer wavelengths the $\mathrm{QE}$ decreases below 5\%. Including optical coupling losses in our setup, we achieve an effective streak camera QE of only $2 \%$ to $3 \%$. Accordingly, the sensitivity of the SPAD is already comparable to our streak camera, and we expect that we can improve it by over an order of magnitude by increasing the fill factor.

The exceptional time resolution of the SPAD is one of the primary characteristics that makes this technology competitive for time-resolved Raman, where fluorescence lifetimes are often as short as nanoseconds in minerals [12]. Our streak camera affords greater time resolution $(\sim 20 \mathrm{ps})$ as well as the ability to collect the complete time evolution in a single image. However, using the present SPAD chip, $200 \mathrm{ps}$ time resolution has been demonstrated for on-chip FLIM [11]. A median FWHM instrument response function of this SPAD array is 230 ps over the entire array, which provides sufficient time resolution when combined with a $500 \mathrm{ps}$ pulse width laser. In this work, due to limitations related to synchronization between the single-pixel SPAD trigger chip and the $128 \times 128$ SPAD array, we were only able to demonstrate a minimum of $32 \mathrm{~ns}$ gate. However, this is not a fundamental limitation, and our next chip design will accommodate faster gating.

We demonstrate the performance of the SPAD array for time-resolved Raman spectroscopy using two 


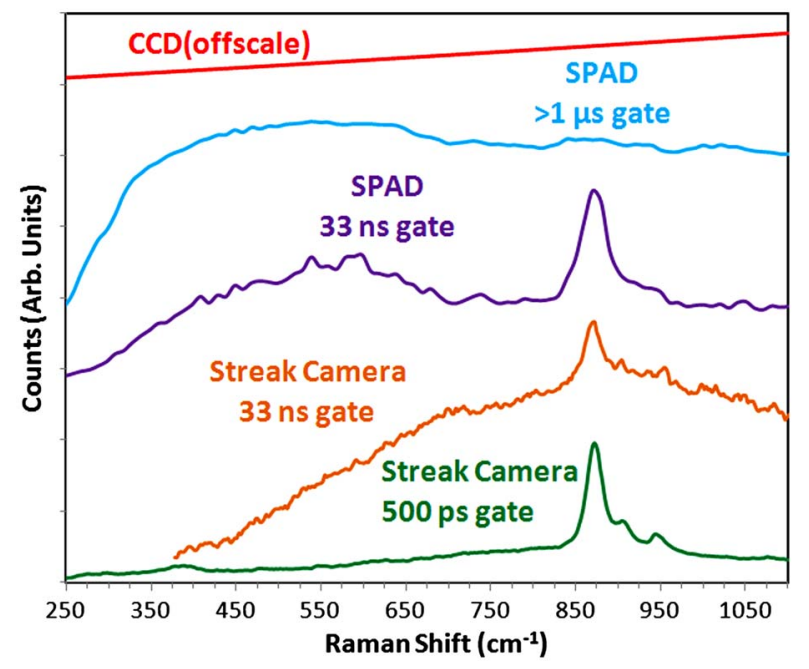

Fig. 3. (Color online) Comparison of willemite Raman spectra using a CCD, streak camera, and $128 \times 128 \mathrm{SPAD}$. The gate time is the time after the start of the laser pulse over which the return signal is averaged. The spectrum obtained with the CCD (CW Raman) is overwhelmed by fluorescence, and the streak camera and SPAD yield similar results with clear Raman spectra.

highly fluorescent natural mineral samples-willemite (Figs. 1 and 3) and spodumene (Fig. 4). Both of these samples, when measured using a CW Raman spectrometer with a $514 \mathrm{~nm}$ laser and CCD detector, contained large fluorescence signals, which saturated the detector, making Raman impossible to obtain. Willemite and spodumene are both well known for their long-lifetime intense luminescence related to $\mathrm{Mn}^{2+}$ impurities [13]. In both cases we acquired spectra using a short gate (33 ns) and a longer gate $(>1 \mu \mathrm{s})$. The short gate is necessary to detect Raman signatures in both of these samples. Results would be improved even further for shorter gate times. Figure 3 compares the results for willemite using a CCD, streak camera, and SPAD. The CCD was saturated over the entire range due to fluorescence. The SPAD and streak camera results are comparable when taken over the same time scale (33 ns). As expected, the streak camera results with a shorter effective gate time $(500 \mathrm{ps})$ show better signal to noise. In all cases, these spectra have not been calibrated for intensity variation versus wavelength, and fixed pattern noise from the detectors has not been subtracted out. The streak camera data were obtained with a high-resolution Raman grating and therefore show better spectral resolution than the SPAD data, which were obtained using a low-resolution broadband grating. This is not however a fundamental characteristic of the detector.

This work has demonstrated the feasibility of obtaining high-sensitivity time-resolved Raman spectra using an SPAD array as an alternative to a streak camera. These results have implications for future planetary in situ instruments as well as numerous other applications such

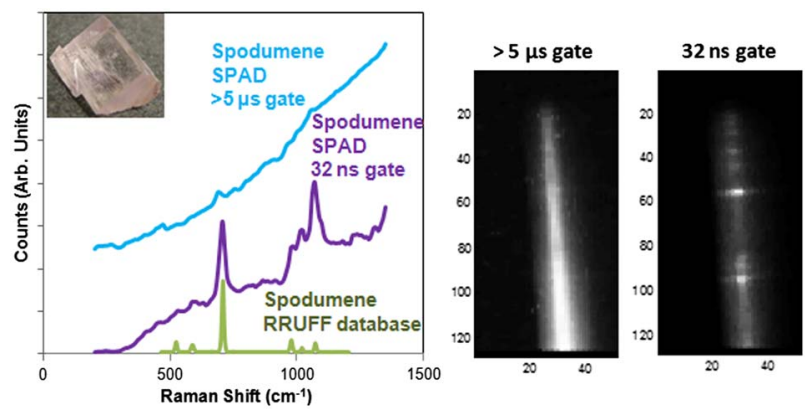

Fig. 4. (Color online) Comparison of spodumene Raman spectra with gate time $32 \mathrm{~ns}$ and $>5 \mu \mathrm{s}$. A spectrum from the RRUFF database (taken at $785 \mathrm{~nm}$ to avoid fluorescence) is shown for reference. The spectra are offset for clarity.

as geosciences, medicine, pharmaceuticals, and materials science. Future work will focus on improvements in fill factor, microlens efficiency, array format, and time resolution. The improved SPAD array will be used for further study on planetary analog minerals.

The research described in this publication was carried out at the Jet Propulsion Laboratory, California Institute of Technology, under a contract with the National Aeronautics and Space Administration (NASA). SPAD development was performed at Delft University of Technology, and CW Raman measurements at Caltech.

\section{References}

1. L. A. Nafie, J. Raman Spectrosc. 41, 1276 (2010).

2. R. Richards-Kortum and E. Sevick-Muraca, Annu. Rev. Phys. Chem. 47, 555 (1996).

3. K. Kneipp, H. Kneipp, I. Itzkan, R. R. Dasari, and M. S. Feld, Chem. Rev. 99, 2957 (1999).

4. P. Vandenabeele, H. G. M. Edwards, and L. Moens, Chem. Rev. 107, 675 (2007).

5. N. Tarcea, T. Frosch, P. Rösch, M. Hilchenbach, T. Stuffler, S. Hofer, H. Thiele, R. Hochleitner, and J. Popp, Space Sci. Rev. 135, 281 (2008)

6. G. B. Courreges-Lacoste, B. Ahlers, and F. R. Perez, Spectrochim. Acta A 68, 1023 (2007).

7. A. Wang, B. L. Jolliff, and L. A. Haskin, "Investigating surface mineralogy, alteration processes, and biomarkers on Mars using laser Raman spectroscopy," presented at the Sixth International Conference on Mars, Pasadena, Calif., July 20-25, 2003, abstract no. 3270 .

8. J. Blacksberg, G. Rossman, and A. Gleckler, Appl. Opt. 49, 4951 (2010).

9. S. K. Sharma, A. K. Misra, S. M. Clegg, J. E. Barefield, R. C. Wiens, and T. Acosta, Phil. Trans. R. Soc. A 368, 3167 (2010), and references therein.

10. L. Carrara, C. Niclass, N. Scheidegger, H. Shea, and E. Charbon, in IEEE International Solid-State Circuits Conference (IEEE, 2009), p. 40.

11. Y. Maruyama and E. Charbon, in Proceedings of the Transducers'11 Conference (IEEE, 2011), p. 1180.

12. M. Gaft and L. Nagli, Eur. J. Mineral. 21, 33 (2009).

13. M. Gaft, R. Reisfeld, and G. Panczer, Modern Luminescence Spectroscopy of Minerals and Materials (Springer, 2005). 